\title{
G somatem \\ Identification of Five m6A-Relevant mRNAs \\ Signature and Risk Score for The Prognostication of Gastric Cancer
}

\section{Dongwei Sun}

Yangzhou University Affiliated Northern Jiangsu People's Hospital: Northern Jiangsu People's Hospital

\section{Xiaorong Hang}

Yangzhou University Affiliated Northern Jiangsu People's Hospital: Northern Jiangsu People's Hospital Jun Ren

Yangzhou University Affiliated Northern Jiangsu People's Hospital: Northern Jiangsu People's Hospital Qiannan Sun

Yangzhou University Affiliated Northern Jiangsu People's Hospital: Northern Jiangsu People's Hospital

\section{Zhu Liu}

Yangzhou University Affiliated Northern Jiangsu People's Hospital: Northern Jiangsu People's Hospital

\section{Yong Wang}

Yangzhou University Affiliated Northern Jiangsu People's Hospital: Northern Jiangsu People's Hospital

\section{Daorong Wang ( $\nabla$ daorong666@sina.com )}

Yangzhou University Affiliated Northern Jiangsu People's Hospital: Northern Jiangsu People's Hospital

\section{Research}

Keywords: Gastric cancer, N6-methyladenosine(m6A), HNRNPC

Posted Date: May 24th, 2021

DOl: https://doi.org/10.21203/rs.3.rs-489055/v1

License: (c) (i) This work is licensed under a Creative Commons Attribution 4.0 International License. Read Full License 


\section{Abstract}

Introduction: N6-methyladenosine (m6A) is the most abundant form of methylation modification in eukaryotic cell mRNA. However, the role of m6A in gastric cancer is unclear, which is one of the most common gastrointestinal malignancies. The m6A-relevant mRNA signatures and risk scores are determined to predict the prognosis of gastric cancer in this study.

Methods: The expression profiles and clinical information of 367 patients were downloaded from the Cancer Genome. Cluster analysis and univariate Cox analysis were used to identify the regulatory factors of RNA methylation associated with gastric cancer prognosis. The co-expression network was constructed by the WGCNA package in R. Then, the correlations between module eigengenes and clinical traits were calculated to identify the relevant modules. We used univariate Cox analysis to screen for genes that were significantly associated with prognosis in the module $(P<0.01$ was considered significant). We identified hub genes by LASSO and multivariate analysis, and developed a Cox prognostic model. Finally, the hub gene expression values weighted by the coefficients from the LASSO regression generated a risk score for each patient, receiver operating characteristic (ROC) and KaplanMeier curves were used to assess the prognostic capacity of the risk scores.

Results: HNRNPC was shown as $\mathrm{P}<0.05$ and $\mathrm{HR}>1$ in the TCGA gastric cancer data set, which might be a pathogenic factor affecting the prognosis of gastric cancer. The results indicated that AARD, ASPN, SLAMF9, MIR3117 and DUSP1 were hub genes affecting the prognosis of gastric cancer patients, and the m6A methylation of these mRNAs might be regulated by HNRNPC. The risk score $=-$ $(0.166195281 \times$ AARD + 0.016850602×ASPN + 0.591607997×SLAMF9 + 0.591607997×MIR3117 + $0.00276337 \times$ DUSP1), and our results indicated a bad performance of the five-gene signature for survival prediction $(\mathrm{P}<0.05)$.

Conclusion: HNRNPC (a m6A RNA methylation regulator) can participate in the serious progression of gastric cancer by regulating the m6A of AARD, ASPN, SLAMF9, MIR3117 and DUSP1, which may be used for prognosis stratification and treatment strategy formulation.

\section{Introduction}

Global cancer statistics have shown that gastric cancer (GC) is considered one of the most invasive cancers and the third leading cause of tumor-related deaths ${ }^{[1]}$. Over the past few decades, various strategies have been developed for GC treatment, which is a significant improvement in the early diagnosis and treatment of $\mathrm{GC}^{[2,3]}$. However, due to the atypical and insidious nature of early clinical symptoms of gastric cancer, only a small number of patients can be clearly diagnosed, while more than $60 \%$ of patients already have local or distant metastasis at the time of diagnosis ${ }^{[4]}$. Therefore, there is an urgent need to develop an effective and effective strategy for early diagnosis and treatment of GC.

N6-methyladenosine ( $\mathrm{m} 6 \mathrm{~A}$ ) modification is the methylation of the adenosine base at the nitrogen-6 position of the mRNA. It is a rich nucleotide modification first discovered in eukaryotic messenger RNA in 
$1974^{[5,6]}$. There are three types of enzymes modified m6A subject to regulation namely writers (methyltransferases, including WTAP, KIAA1429, RBM15 and METTL3/14), readers (YTH domain containing RNA binding proteins and heterogeneous nuclear ribonucleoprotein, including YTHDF1/2/3, YTHDC1 and HNRNPC) and erasers (demethylases, including ALKBH5 and FTO) ${ }^{[7,8]}$. In recent years, it has been found that the expression of HNRNPC is related to the development of malignant tumors and gliomas, is involved in the occurrence of glioblastoma multiforme which can predict the prognosis ${ }^{[9]}$. HNRNPC was proved to promote oral squamous cell carcinoma carcinogenesis and can be an independent biomarker prognostic biomarker ${ }^{[10]}$. Studies have also shown the potential value of HNRNPC as a prognostic and therapeutic marker for $\mathrm{GC}$, and it plays an important role in promoting the translation of human gastric cancer genes ${ }^{[11]}$. In this study, we downloaded the expression profile and clinical data of the Tumor Genome Atlas (TCGA). By using the univariate Cox analysis method, we determined the prognostic m6A RNA methylation regulator. In addition, we established weighted gene co-expression network analysis (WGCNA), lasso regression analysis (least absolute shrinkage and selection operator) and multivariate COX analysis to identify the pivotal genes that might be regulated by m6A RNA methylation regulators and related to the prognosis of GC. Finally, according to the selected combination of pivot genes, a risk scoring model was constructed to evaluate its application in the prognosis of GC. These hub genes are closely related to the methylation m6A RNA methylation regulators, which provides new ideas for the research of GC.

\section{Methods}

\subsection{Patient datasets and $\mathrm{m} 6 \mathrm{~A}$ regulators}

The mRNA expression data and corresponding clinical information of patients with gastric cancer were downloaded from The Cancer GenomeAtlas (TCGA). This study included the expression profiles of 309 patients with complete follow-up data in the TCGA database. Tcgabiollinks package was used to download TCGA data.

In this study, we included many m6A methylation regulators, such as writers : RNA-binding motif 15 (RBM15), KIAA1429, METTL3, METTL14, zinc finger CCCH domain-containing protein 13 (ZC3H13) and WT1-associated protein ; readers : YTH m6A RNA-binding protein 1 (YTHDF1), YTH m6A RNAbinding protein 2 (YTHDF2), YTH domain-containing 1 (YTHDC1), YTH domain-containing 1 (YTHDC2), and heterogeneous nuclear ribonucleoprotein C (HNRNPC); and erasers : a-ketoglutaratedependent dioxygenase alkB homolog 5 (ALKBH5) and fat massand obesity-associated protein (FTO). In order to study the differential expression of m6A RNA methylation regulators in tumor and normal tissues, we analyzed the mRNA expression profile of TCGA-gastric cancer (including 58 normal samples and 309 tumor samples). Cluster analysis was applied to m6A RNA methylation regulators, and heatmaps and violin maps were presented to show differences. The pheatmap $\mathrm{R}$ package and the vioplot $\mathrm{R}$ package were used for drawing the plots. In addition, we used univariate Cox analysis to identify m6A-related 
genes related to the prognosis of gastric cancer (m6A regulatory genes with value $<0.05$ considered statistically significant).

\subsection{Co-expression network construction and identification of clinically significant modules}

The co-expression network was constructed by the WGCNA package in $\mathrm{R}^{[12]}$. The genes with variances greater than all variance quartiles were selected and those genes with larger variances and larger mean variations in different samples were considered. The expression data profile of the selected genes was qualified and the samples were clustered to detect outliers. Gene clustering modules were identified on the basis of the clinical features (including the expression of the m6A regulatory genes that we selected before) and topological overlap matrixbased dissimilarity ${ }^{[13]}$. Next, the relevance between clinical features and module eigengenes was used to identify the correlated modules. Highly correlated modules were considered to be very significant for our research.

\subsection{Identification of hub-genes and riskscore model construction}

We selected the modules of interest where the genes in the modules were defined as highly relevant with certain clinical traits. Next, Univariate Cox analysis and LASSO were used to screen for the genes that were significantly correlated to prognosis in the module ( $P<0.01$ was considered significant). Cluster Profiler R package was used for Gene Ontology (GO) and Kyoto Encyclopedia of Genes and Genomes (KEGG) enrichment analyses of screened genes, and $P<0.05$ was considered a statistically significant difference. The TCGA samples were randomly divided into two groups: 153 samples were tested, and 156 samples were verified. There was no statistically significant difference in the expression of HNRNPC and other clinicopathological variables between the two groups. In the training set $(n=156)$, LASSO regression was perfomed to screen for gastic cancer prognosis-related genes based on lambda.min (the lambda corresponding to the smallest mean error), and the hub genes were selected. LASSO was analyzed with the "glmnet" package in R. The hub gene expression values weighted by the coefficients from the LASSO regression generated a risk score for each patient. Finally, The Survminer R package was used to find the optimal cutoff for the risk score, while receiver operating characteristic (ROC) and KaplanMeier curves were used to assess the prognostic capacity of the risk scores.

\section{Results}

\subsection{Identification of prognostic m6A RNA methylation regulators}

Univariate Cox analysis was performed to identify the gene map of m6A related to the prognosis of liver cancer patients (forest) to show m6A regulators with $\mathrm{P}<0.05$. Based on the univariate COX analysis, we found that the high expression of HNRNPC is better related to the prognosis of the following patients: HNRNPC of gastric cancer in the TCGA data set shows $P<0.05$, and $H R>1$, which can be considered as pathogenic factors negatively affecting the prognosis of gastric cancer (Figure $1 \mathrm{~A}$ ).

\subsection{Differential expression of m6A RNA methylation regulators}


In the TCGA set, 58 cases were normal samples and 309 cases were tumor samples. Heatmaps and violin maps were drawn according to the different gene expression levels. According to the results, we can conclude that HNRNPC had higher expression in the tumor samples than in the normal samples. RBM15, WTAP, METTL3, YTHDF2, YTHDF1, YTHDC1, YTHDC2, KIAA1429, ZC3H13 and HNRNPC were shown significantly higher expression in tumors than in normal tissues (Figure 1B). As shown in the violin plot (Figure 2), the expression of YTHDC2, RBM15, ZC3H13, METTL3, YTHDC1, KIAA1429, WTAP, YTHDF1, YTHDF2 HNRNPC in normal tissues is obviously different from that in tumor tissues, and the differences had significance $(P<0.05)$. They showed higher expression in tumor tissues.

\subsection{Co-expression network construction}

As mentioned above, this study calculated the variance of the expression of each gene in all samples, and taking the variance value greater than the quartile as the standard, a total of 6685 genes were screened out. A hierarchical clustering tree was constructed from 6685 genes in 309 tumor samples. Then, 309 samples and sample clinical information were hierarchically clustered (Figure 3A). To construct a scale-free network, there was the need to choose the appropriate weighting factor $\beta$ while moderately retaining the average connectivity of each gene node. We finally chose $\beta=5$ to construct the co-expression network (Figure 3B). After determining the $\beta$ value, a total of 15 modules were identified (Figure 3C).

\subsection{Correlation between modules and phenotypes}

According to the correlation between each module and the clinical phenotype, we selected the modules that were significantly associated with prognosis and HNRNPC expression. The turquoise and magenta modules were significantly highly associated with HNRNPC expression (positive values indicate a positive correlation, negative values indicate a negative correlation) and had a stronger correlation with the pathologic stage. This indicates that the genes in the two modules may be regulated by HNRNPC and play a role in the prognosis of gastric cancer patients (Figure 4).

\subsection{Hub genes identification}

In order to further determine the prognostic genes regulated by HNRNPC, we chose the turquoise and magenta modules to conduct further research on 1538 genes. A preliminary selection of prognostic genes was made by univariate Cox, where a $\mathrm{P}<0.05$ was used as a cutoff for screening prognostic genes, and 98 genes were selected. The 98 selected genes were analyzed by clusterProfiler R package for $\mathrm{GO}$ and KEGG pathway analysis. In the biological process terms of GO analysis, the genes were mainly enriched in "positive regulation of ERK1 andERK2 cascade," "cellular response to chemokine," "monocyte chemotaxis," "regulation of cartilage development," "regulation of phospholipase activity," "programmed cell death involved in cell development," "chemokine-mediated signaling pathway." In cell component terms, Differentially Expressed Genes (DEGs) were mainly enriched in "collagen-containing extracellular 
matrix," "basement membrane," "fibrillar center." In molecular function terms, DEGs were mainly enriched in "extracellular matrix structural constituent," "G protein-coupled receptor binding,"

"endodeoxyribonuclease," "activity, producing 5'-phosphomonoesters," "chemokine receptor binding,"

"extracellular matrix structural constituent conferring compression resistance." KEGG pathway analysis demonstrated that 98 selected genes were significantly enriched in "endonuclease activity, active with either ribo-or deoxyribonucleic acids and producing 5'-phosphomonoesters," "extracellular matrix structural constituent," "titin binding," "endodeoxyribonuclease," "activity, producing 5'-phosphomonoesters," "extracellular matrix structural constituent conferring compression resistance," "endonuclease activity," "nuclease activity," "transmembrane receptor protein kinase activity" and "dioxygenase activity," etc (Figure 5).

Then, 309 TCGA samples were randomly divided into a training set and an testing set. The tableone $\mathrm{R}$ package was used to describe the clinical information difference between the internal training set and the internal testing set. The results showed that expression of HNRNPC and the other clinicopathological variables was not significantly different between the two groups (Table 1).

Table 1 There was no significant difference between the two groups of clinical phenotypes.

\begin{tabular}{|c|c|c|c|}
\hline & Training set & Testing set & $\mathbf{P}$ value \\
\hline $\mathrm{n}$ & 156 & 153 & \\
\hline age $₫ 65 \rrbracket \% \rrbracket$ & 85 & 101 & 0.494 \\
\hline gender=male $(\%)$ & 89 & 101 & 0.106 \\
\hline grade & & & 0.257 \\
\hline 1 & 2 & 3 & \\
\hline 2 & 53 & 60 & \\
\hline 3 & 101 & 90 & \\
\hline stage & & & 0.098 \\
\hline ૫ & 24 & 21 & \\
\hline ૫ & 56 & 44 & \\
\hline ૫ & 64 & 68 & \\
\hline ૫ & 12 & 20 & \\
\hline 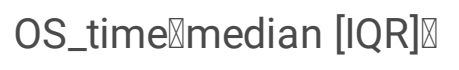 & $404.5[0,3720]$ & $383[0,2171]$ & 0.273 \\
\hline OS_event=0/1(\%) & $106 / 50$ & $96 / 57$ & 0.337 \\
\hline HNRNPC(mean(SD)) & $39.94(9.98)$ & $39.35(10.51)$ & 0.507 \\
\hline
\end{tabular}


In the experimental group, a total of 98 prognostic genes were screened for the two modules using LASSO analysis and multivariate COX analysis. The results showed that AARD, ASPN, SLAMF9, MIR3117 and DUSP1 are real hub genes that are associated with patient prognosis (Figure 6A-C). In the TCGA datasets, a significant correlation was found between the expression of HNRNPC and that of the hub genes (Figure 6D).

\subsection{Risk scores}

Five genes were identified and subsequently used to construct a prognostic gene signature. The risk score $=-(0.166195281 \times$ AARD $+0.016850602 \times$ ASPN

$+0.591607997 \times$ SLAMF9 + 0.591607997×MIR3117 + 0.00276337×DUSP1), and we used the Survminer R package to find the optimal cutoff for the risk score, while ROC and Kaplan-Meier curves were used to assess the prognostic ability of the risk scores. We plotted the risk score distribution, the time-dependent ROC curve and the survival analysis of the training set and testing set (Figure 8 ). Our results indicated a bad performance of the five-gene signature for survival prediction $(P<0.05)$.

\section{Discussion}

N6 methyladenosine $(\mathrm{m} 6 \mathrm{~A})$ modification is the most common modification in human mRNA ${ }^{[14]}$, which is considered to be a new epigenetic regulator of mRNA processing and translation. Increasing studies have revealed that the maladjustment of $\mathrm{m} 6 \mathrm{~A}$ is closely related to abundant physiological and pathological phenomena, including carcinogenesis ${ }^{[15]}$, obesity, immune maladjustment and so on ${ }^{[16,17]}$. In recent years, Mounting evidence has proved that m6a-related genes play a vital role in the genesis and the development of gastic cancer ${ }^{[2,18]}$. For example, Lin S, et al reported that METTL3 inhibits the mobility and proliferation of human gastric cancer cells and leads to inactivation of the AKT signaling pathway, indicating that it may be a meaningful and potential target for the treatment of human gastric cancer ${ }^{[19]}$. It has been found that the transfer of HNRNPC location may be related to chemoresistance of gastric cancer, suggesting the potential usefulness of HNRNPC as a prognostic and therapeutic marker of GC ${ }^{[11]}$. $\mathrm{Pi}$ J, et al reported that YTHDF1 directly promotes the translation of the key Wnt receptor frizzled7 (FZD7) in an m6a-dependent manner, so that the mutant YTHDF1 enhances the expression of FZD7, which ultimately leads to the over-activation of the $\mathrm{Wnt} / \beta$-catenin pathway and promotes the occurrence of $\mathrm{GC}^{[20]}$.

In this study, we first evaluated the expression of HNRNPC in GC and found that the expression of HNRNPC in tumor samples increased significantly. As an effective IRES activator, HNRNPC is related to the establishment and maintenance of malignant phenotype. It is regulated by increasing the level of IGG1R and ultimately promotes the occurrence of gastric cancer ${ }^{[11,21]}$. As an important m6A methyltransferase, HNRNPC has been found to play a very important and potential role in a variety of 
physiological and biochemical functions, and it is also related to the occurrence and development of many cancers.

We finally identified five hub genes (AARD, ASPN, SLAMF9, MIR3117 and DUSP1) that may be regulated by HNRNPC. In previous studies, these five genes have been found to be involved in the development of many diseases. ASPN promotes the migration and invasion of colorectal cancer cells via TGF$\beta / \mathrm{Smad} 2 / 3$ pathway and could serve as a potential prognostic biomarker in colorectal cancer patients ${ }^{[22]}$. It has been found that ASPN promotes cell proliferation by interacting with PSMD2, and down-regulation of its effectors and serves as a potential therapeutic target in $\mathrm{GC}^{[23]}$. The expression of SLAMF9 in melanocyte lesions may indicate genetic susceptibility to the development of malignant melanoma, which suggests that SLAMF9 plays an important role in melanoma biology ${ }^{[24]}$. It has been reported that miR-3117 participates in the proliferation of HepG2 by targeting PHLPPL, thereby participating in the occurrence and development of liver cancer ${ }^{[25]}$. The elevated DUSP1 expression is related to tumor progression, drug resistance and poor prognosis, and can be used as a predictive biomarker for apatinib treatment ${ }^{[26]}$. However, there are few studies on AARD. Our research indicated that there is a certain connection between these five hub genes and HNRNPC, however, this remains to be verified by further experiments.

\section{Conclusion}

Our research revealed a risk model consisting of five m6A-relevant genes, which may be useful for the prediction and diagnosis of gastric cancer. In addition, this discovery also provides motivation for basic medical research on $\mathrm{m} 6 \mathrm{~A}$ methylation in gastric cancer.

\section{Abbreviations}

m6A: N6-methyladenosine; GC: Gastric cancer; TCGA: The Cancer Genome Atlas; OS: overall survival; TNM: tumor node metastasis; FTO: Fat Mass and Obesity associated protein; WTAP: Wilms tumor 1 associated protein; ALKBH5: alkB homolog 5; $\mathrm{ZC} 3 \mathrm{H} 13$ : zinc finger $\mathrm{CCCH}$ domain-containing protein 13; METTL3: Methyltransferase like 3; METTL14: Methyltransferase like 14; HNRNPC: Heterogeneous nuclear ribonucleoprotein C (C1/C2); RBM15: RNA binding motif protein 15; YTHDC1: YTH domain containing 1; YTHDC2: YTH domain containing 2; YTHDF1: YTH N6-methyladenosine RNA binding protein 1; YTHDF2: YTH N6-methyladenosine RNA binding protein 2; ROC: Receiver operating characteristic; WGCNA:

Weighted gene co-expression network analysis; DEGs: Differentially Expressed Genes; AARD: alanine and arginine rich domain containing protein; ASPN: asporin; SLAMF9: The signaling lymphocyte activating molecule family; miRNAs囚microRNAs囚DUSP1区Dual specificity phosphatase 1.

\section{Declarations}

\section{Ethics approval and consent to participate}


The study was approved by the ethical committee of Northern Jiangsu People's Hospital, Yangzhou, China. The study was performed in accordance with the Declaration of Helsinki.

\section{Consent for publication}

Not applicable.

\section{Availability of data and materials}

The data analyzed during the current study are available from the corresponding author on reasonable request.

\section{Competing interests}

The authors declare no conflicts of interest.

\section{Funding}

Not applicable.

\section{Authors' contributions}

Conception and design: Dongwei Sun; (II) Administrative support: Daorong Wang; (III) Provision of study materials: Dongwei Sun, Xiaorong Huang, Qiannan Sun, Daorong Wang; (IV) Collection and assembly of data: Dongwei Sun, Xiaorong Huang, Jun Ren; (V) Data analysis and interpretation: Dongwei Sun, Xiaorong Huang, Zhu Liu; (VI) Manuscript writing: Dongwei Sun, Xiaorong Huang; (VII) Final approval of manuscript: Daorong Wang.

\section{Acknowledgements}

The authors acknowledge the support from the Northern Jiangsu People's Hospital, We also greatly appreciate that Prof. Daorong Wang and Dr. Jun Ren supervised this study.

\section{Authors' Information}

Dongwei Sun\#, Email『subeiwei21@sina.com

Xiaorong Huang\#",Email囚h1991x61r@163.com

Jun Ren, Email|freezingfall@163.com

Qiannan Sun, Email囚18042688505@163.com

Zhu Liu,Email『3231835218@qq.com

Yong Wang, Email『2472394093@qq.com 


\section{References}

1. Freddie B. Jacques, et al.Global cancer statistics 2018: GLOBOCAN estimates of incidence and mortality worldwide for 36 cancers in 185 countries[J].CA: a cancer journal for clinicians,2018.

2. Guan K, Liu X, Li J, et al.Expression Status And Prognostic Value Of M6A-associated Genes in Gastric Cancer[J].Journal of Cancer,2020, 11(10): p. 3027-3040.

3. Wang X, Wang Y, Meng Q, et al.Postoperative chemoradiotherapy in gastric cancer: a phase I study of radiotherapy with dose escalation of oxaliplatin, 5-fluorouracil, and leucovorin (FOLFOX regimen) [J].Medical Oncology,2011, 28(1 Supplement): p. 274-279.

4. Thrift AP, Jove AG, Liu Y, et al.Associations of Duration, Intensity, and Quantity of Smoking With Risk of Gastric Intestinal Metaplasia[J].Journal of Clinical Gastroenterology,2020, publish ahead of print.

5. Niu Y, Zhao X, Wu YS, et al. N6-methyl-adenosine (m6A) in RNA: An Old Modification with A Novel Epigenetic Function[J]. Genomics Proteomics Bioinformatics. 2013;11(1):8-17.

6. Chen K, Wei Z, Zhang Q, et al.WHISTLE: a high-accuracy map of the human N6-methyladenosine (m6A) epitranscriptome predicted using a machine learning approach[J].Nucleic Acids Research,2019(7): p. 7.

7. Wu L, Wu D, Ning J, et al.Changes of N6-methyladenosine modulators promote breast cancer progression[J].BMC Cancer,2019, 19(1).

8. Yang Y, Hsu PJ, Chen YS, et al.Dynamic transcriptomic $m 6$ A decoration: writers, erasers, readers and functions in RNA metabolism[J].Cell Research,2018.

9. Wang LC, Chen SH, Shen XL, et al.M6A RNA Methylation Regulator HNRNPC Contributes to Tumorigenesis and Predicts Prognosis in Glioblastoma Multiforme[J].Frontiers in Oncology,2020, 10.

10. Huang GZ, Wu QQ, Zheng ZN, et al.M6A-related bioinformatics analysis reveals that HNRNPC facilitates progression of OSCC via EMT[J].Aging (Albany NY),2020, 12(12).

11. Hao $\mathrm{H}$, Yong $\mathrm{H}$, Cheng $\mathrm{Z}$, et al.HNRNPC as a candidate biomarker for chemoresistance in gastric cancer[J].Tumor Biology,2016, 37(3): p. 1-8.

12. Langfelder P,Horvath S.WGCNA: an R package for weighted correlation network analysis[J].Bmc Bioinformatics,2008, 9(1): p. 559.

13. Botía J, Vandrovcova J, Forabosco P, et al.An additional k-means clustering step improves the biological features of WGCNA gene co-expression networks[J].Bmc Systems Biology,2017, 11(1).

14. Wei Z, Jing-Zi, et al. Detection of N6methyladenosine modification residues (Review)[J]. International Journal of Molecular Medicine; 2019.

15. Dan, Jin, Jiwei, et al.m < sup $>6</$ sup $>$ A mRNA methylation initiated by METTL3 directly promotes YAP translation and increases YAP activity by regulating the MALAT1-miR-1914-3p-YAP axis to induce NSCLC drug resistance and metastasis[J].Journal of hematology \& oncology,2019, 12(1): p. $135-135$. 
16. Chen T, Hao Y, Zhang Y, et al.M(6)A RNA Methylation Is Regulated by MicroRNAs and Promotes Reprogramming to Pluripotency[J].Cell Stem Cell,2015.

17. Cai X, Wang X, Cao C, et al.HBXIP-elevated methyltransferase METTL3 promotes the progression of breast cancer via inhibiting tumor suppressor let-7g[J].Cancer Letters,2017: p. 1565.

18. Zhang $\mathrm{C}$, Zhang $\mathrm{M}, \mathrm{Ge} \mathrm{S}$, et al.Reduced $\mathrm{m} 6 \mathrm{~A}$ modification predicts malignant phenotypes and augmented Wnt/PI3K-Akt signaling in gastric cancer[J].Cancer Medicine,2019, 8(10).

19. Lin S, Liu J, Jiang W, et al.METTL3 promotes the proliferation and mobility of gastric cancer cells[J].Open medicine (Warsaw, Poland), 14(1): p. 25.

20. Pi J, Wang W, Ji M, et al.YTHDF1 promotes gastric carcinogenesis by controlling translation of FZD7[J].Cancer Research,2020: p. canres.0066.2020.

21. Blume SW, Jackson NL, Frost AR, et al.Northwestern profiling of potential translation-regulatory proteins in human breast epithelial cells and malignant breast tissues: evidence for pathological activation of the IGF1R IRES[J]. Experimental \& Molecular Pathology,2010, 88(3): p. 341-352.

22. Li H, Zhang Z, Chen L, et al.Cytoplasmic Asporin promotes cell migration by regulating TGF$\beta /$ Smad2/3 pathway and indicates a poor prognosis in colorectal cancer[J].Cell Death \& Disease,2019, 10(2).

23. Zhang $\mathrm{Z}$, $\mathrm{Li} \mathrm{H}$, Zhao Y, et al.Asporin promotes cell proliferation via interacting with PSMD2 in gastric cancer[J].Frontiers in Bioscience,2019, 24(6): p. 1178-1189.

24. Claudia D. Julia, et al.The novel immunoglobulin super family receptor SLAMF9 identified in TAM of murine and human melanoma influences pro-inflammatory cytokine secretion and migration[J]. Cell Death \& Disease; 2018.

25. Cui X, Li Q, He Y. miR-3117 regulates hepatocellular carcinoma cell proliferation by targeting. PHLPPL[J].Molecular \& Cellular Biochemistry; 2017.

26. Teng F, Xu Z, Chen J, et al.DUSP1 induces apatinib resistance by activating the MAPK pathway in gastric cancer[J].Oncology Reports,2018.

\section{Figures}




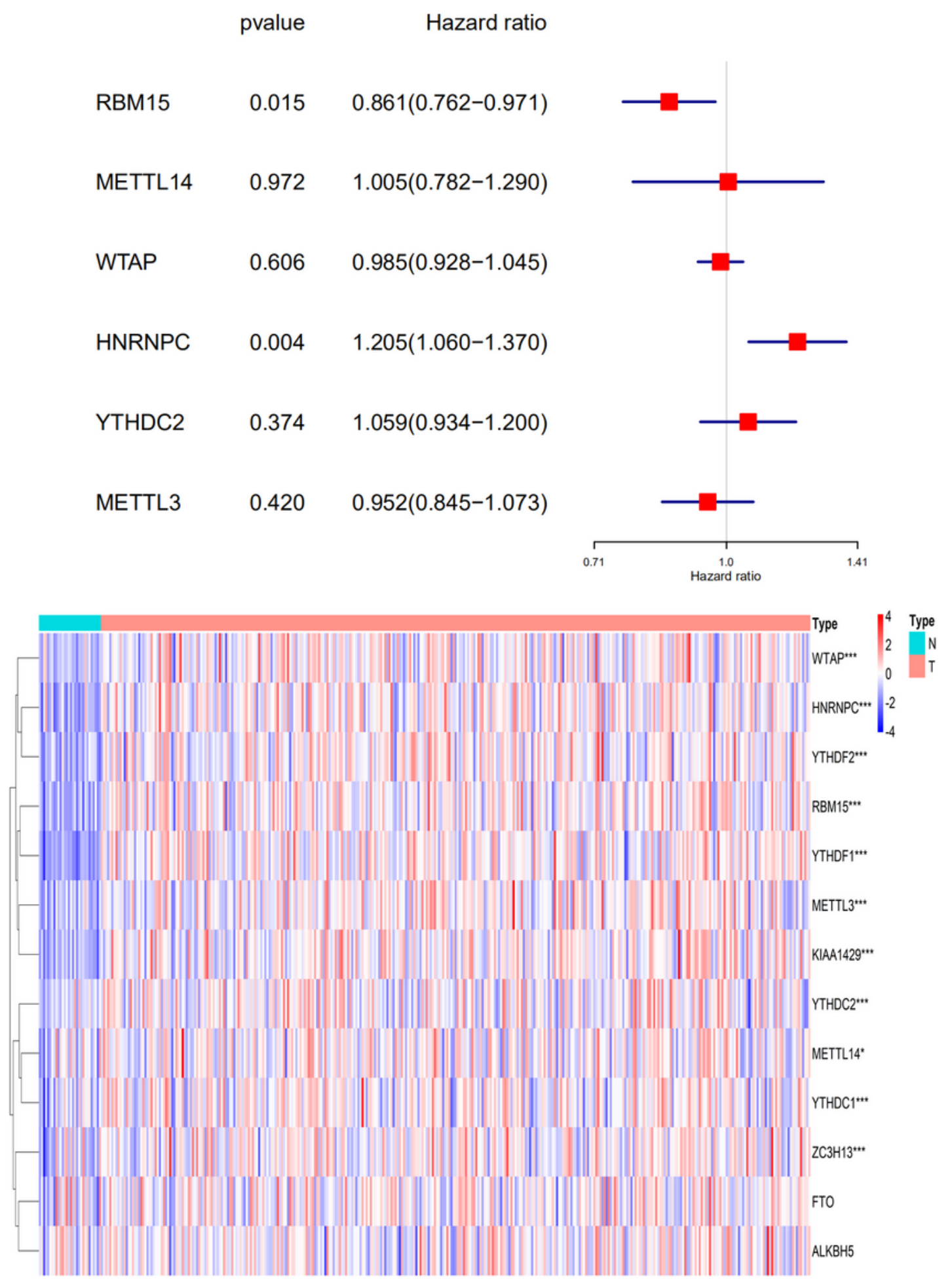

Figure 1

Identification of prognostic m6A RNA methylation regulators. A, Forest plot of univariate Cox analysis in gastric cancer (HNRNPC is related to prognosis and is shown as a pathogenic factors in TCGA). $B$, Heatmap exhibiting the mRNA expression alteration of m6A-associated genes in TCGA datasets. The expression levels of different genes in normal samples and tumor samples are significantly different, and 
the HNRNPC of tumor samples is significantly higher than that of normal samples. N, normal tissue; T, tumor tissue; TCGA, the cancer genome map.

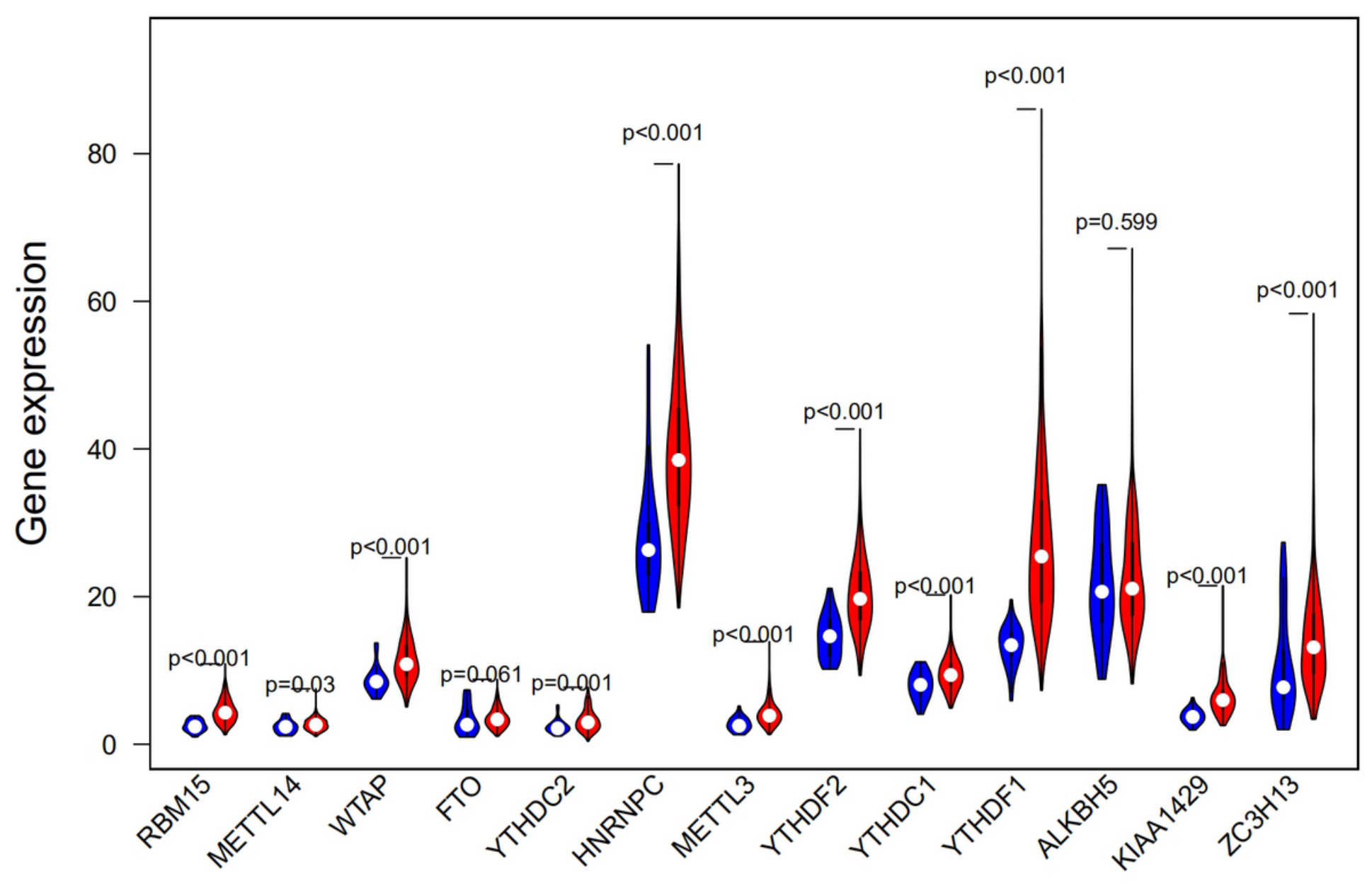

Figure 2

Differential expression of m6A RNA methylation regulators. Vioplot visualizing the differentially m6A RNA methylation regulators in gastric cancer (assume blue is normal and red is gastric cancer). HNRNPC showed higher expression in tumor tissues. 

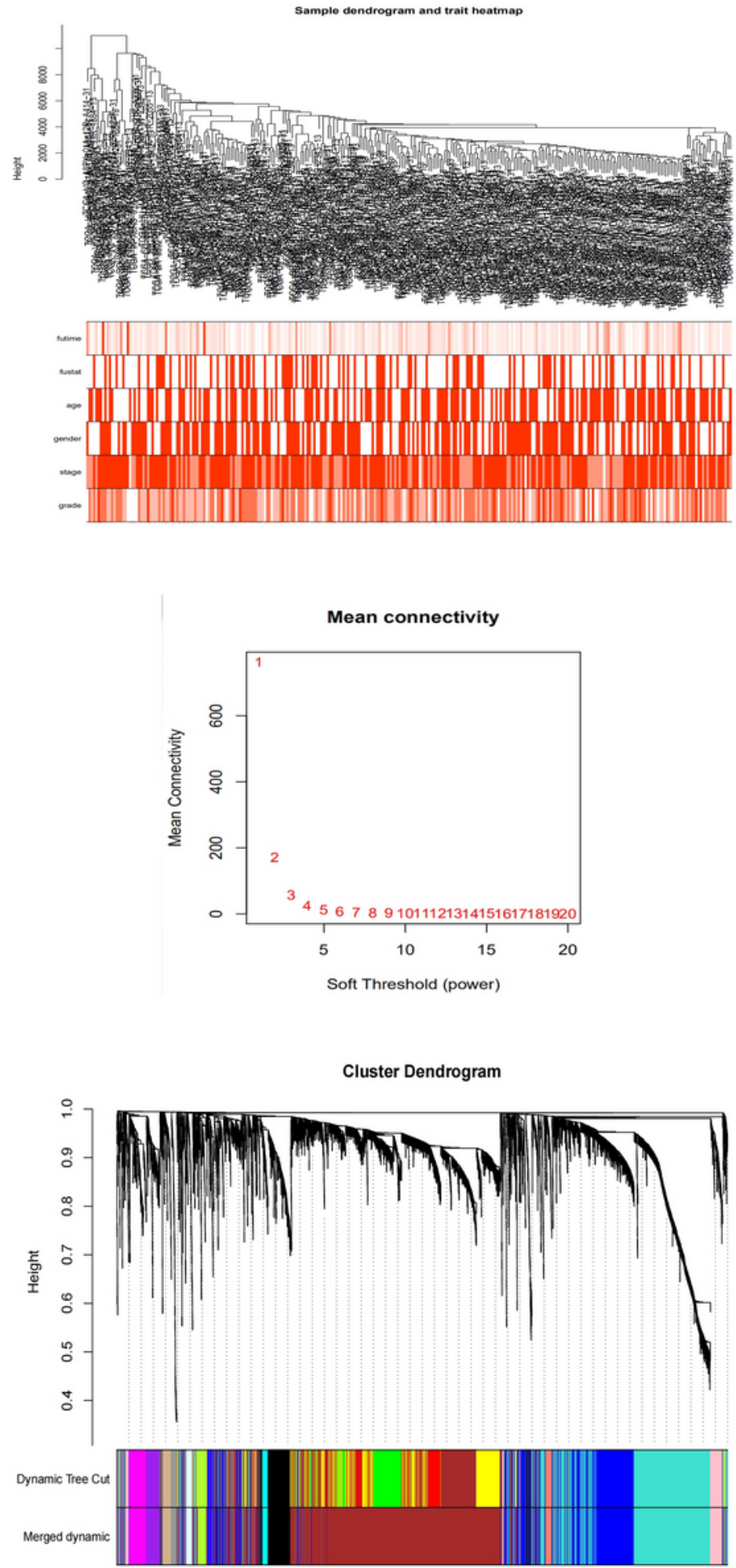

\section{Figure 3}

Co-expression network construction. A, Clustering of 309 tumor samples and clinical information (where the number/stage is larger, the darker color is shown). B, The scale-free index calculated under different $\beta$ and the average connectivity calculated under different $\beta$ (the numbers in the figure indicate the corresponding soft threshold power. The approximate scale-free topology can be achieved at a soft 
threshold power of 5). C, Gene clustering tree diagram. Based on the common topological overlap, each color module represents a module that contains a set of highly connected genes.

\section{Module-trait relationships}

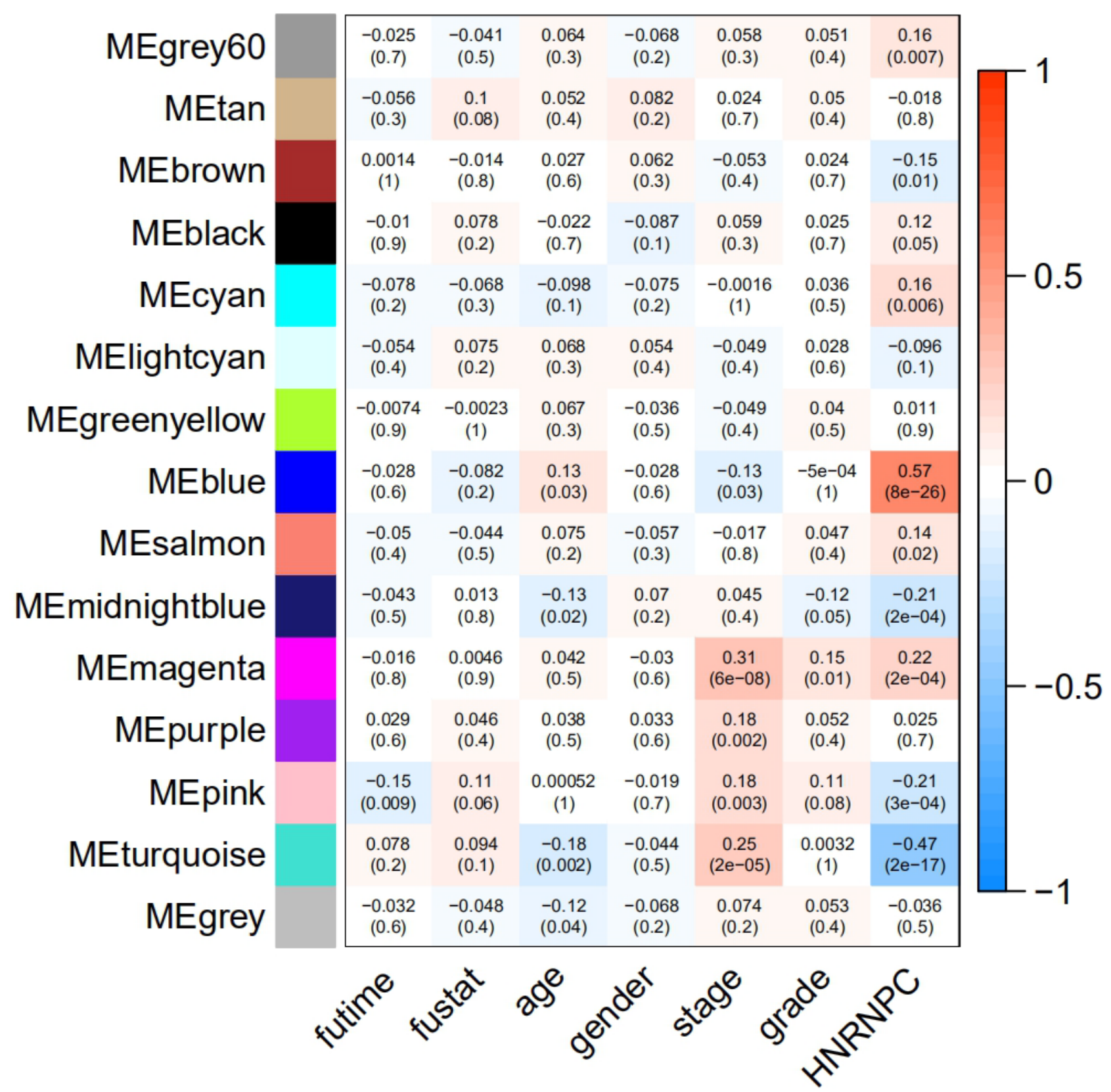

Figure 4

Correlation between modules and phenotypes. Each row in the figure corresponds to a gene module, and each column corresponds to a clinical phenotype. The numbers in brackets indicate the $\mathrm{P}$ value, and the numbers without the brackets indicate the correlation. 

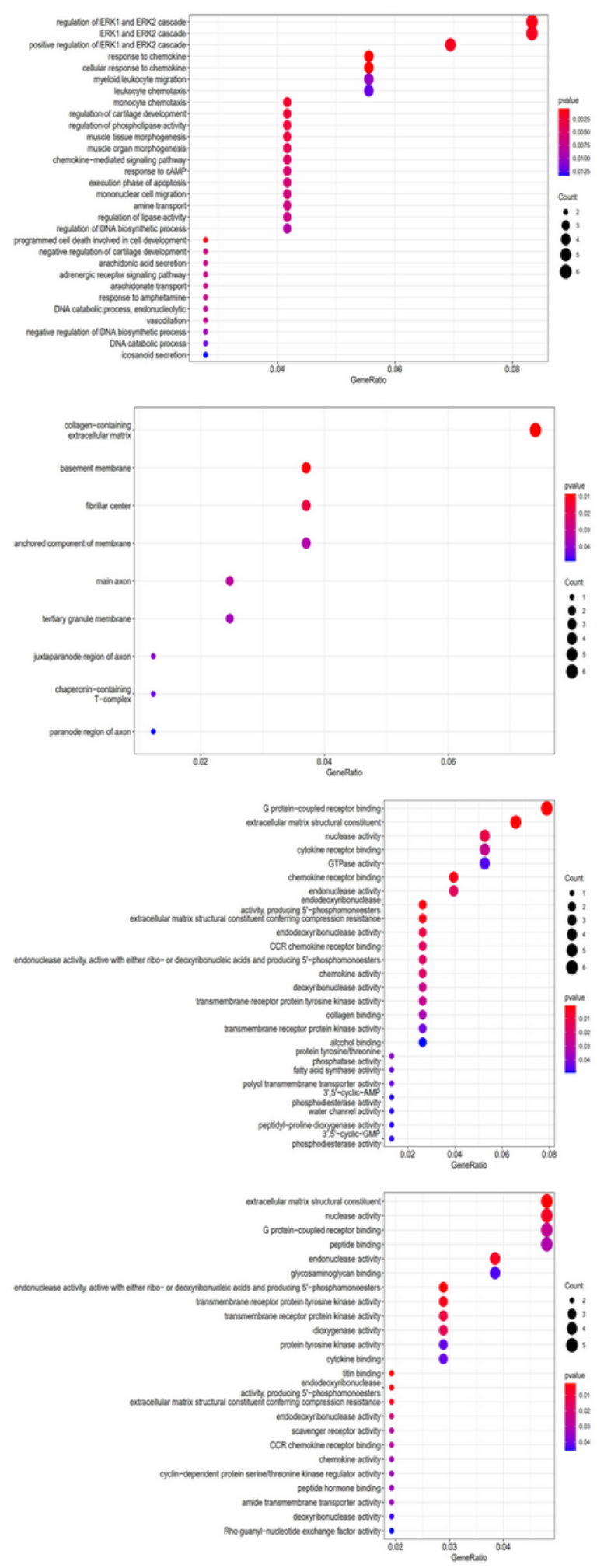

Figure 5

Functional enrichment analysis of 98 selected genes. KEGG, Kyoto Encyclopedia of Genes and Genomes. 

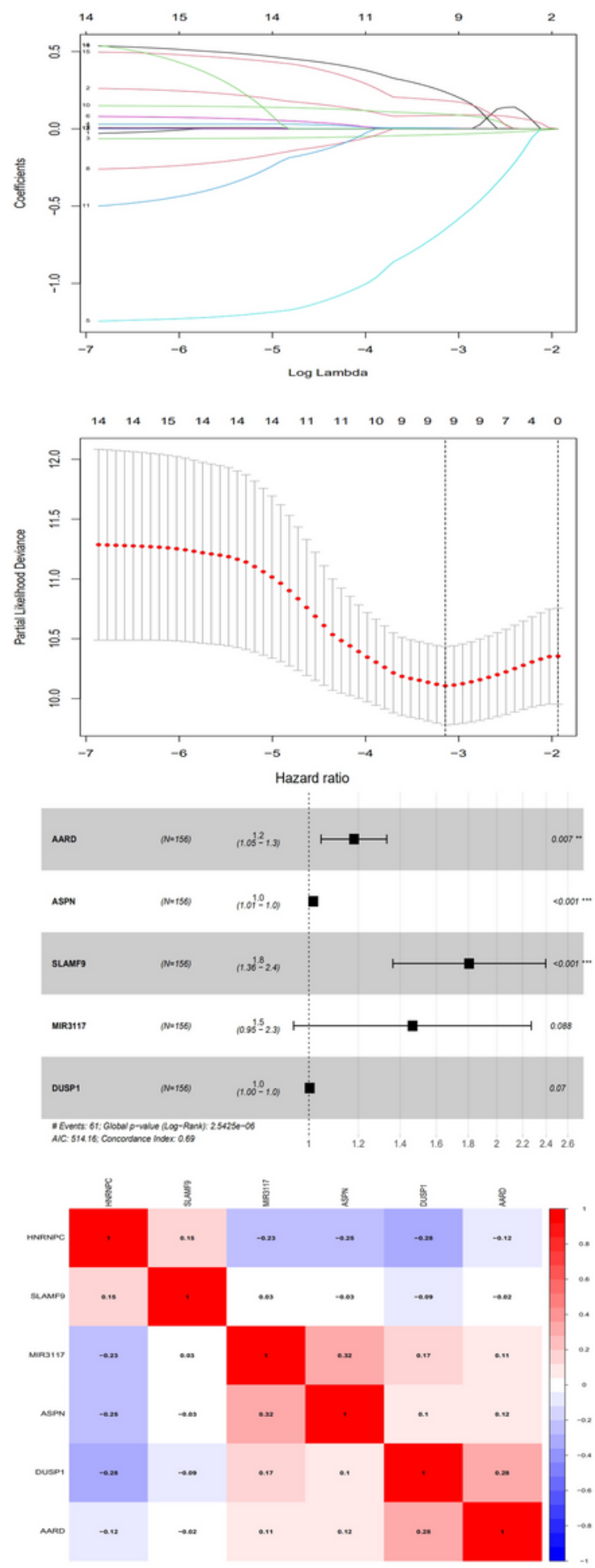

Figure 6

Hub genes identification. A, Distribution of least absolute shrinkage and selection operator (LASSO) coefficients for 98 genes. B, Partial likelihood deviation of the LASSO coefficient distribution. Vertical dashed lines indicate lambda.min and lambda.1se. C,Forest plot of multivariate Cox analysis in gastric cancer. D, Expression of HNRNPC and hub genes showed a significant correlation. TCGA, The Cancer Genome Atlas. 
Survival curve $(p=3.964 \mathrm{e}-04)$

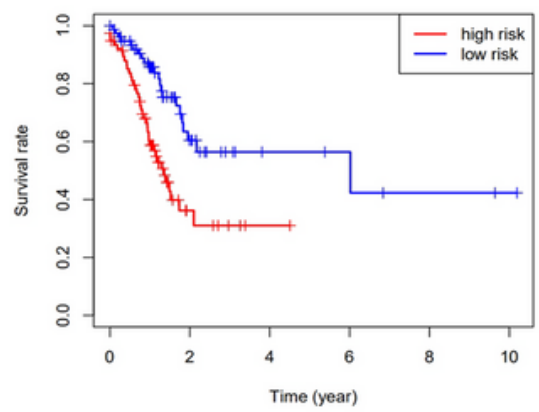

ROC curve $(A U C=0.736)$
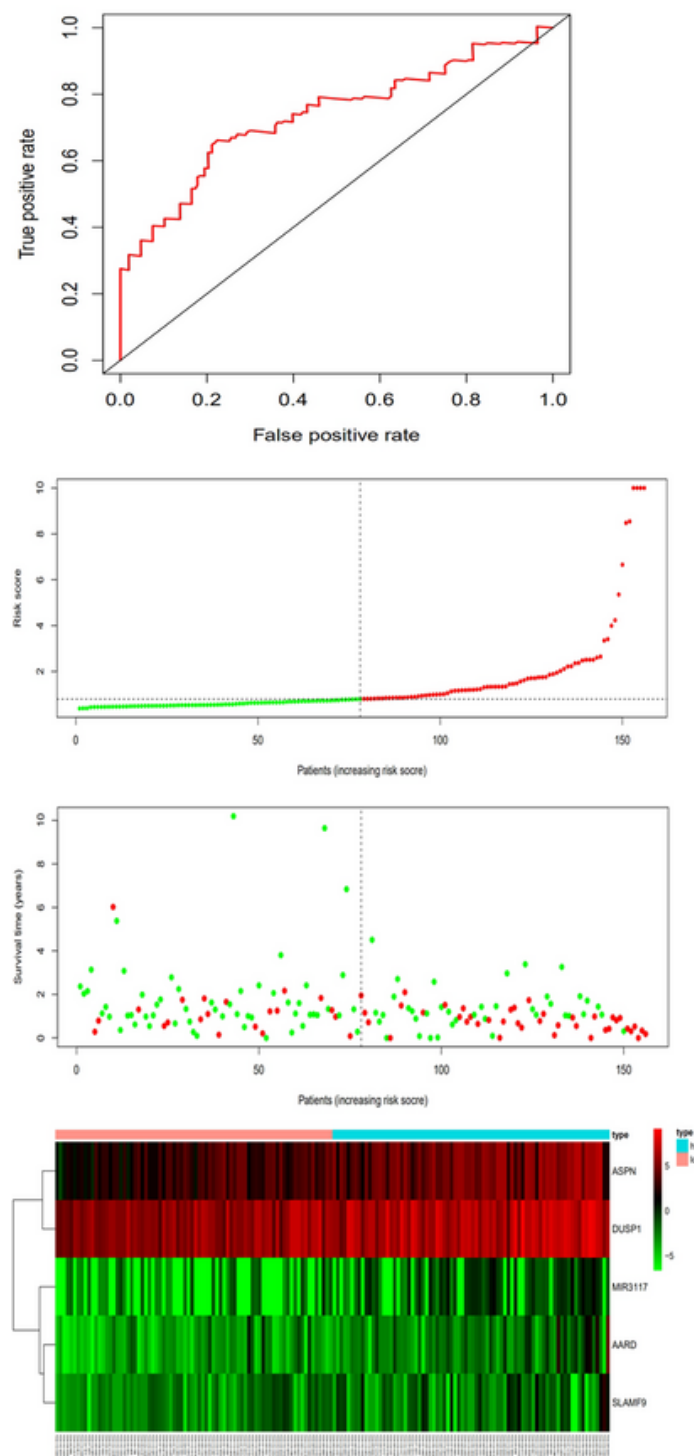

Survival curve $(p=1.186 e-02)$

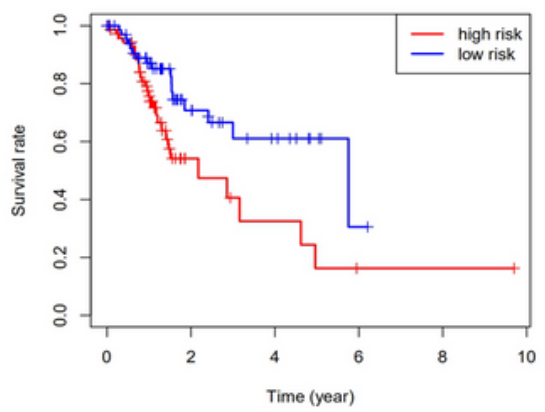

ROC curve $(A U C=0.621)$
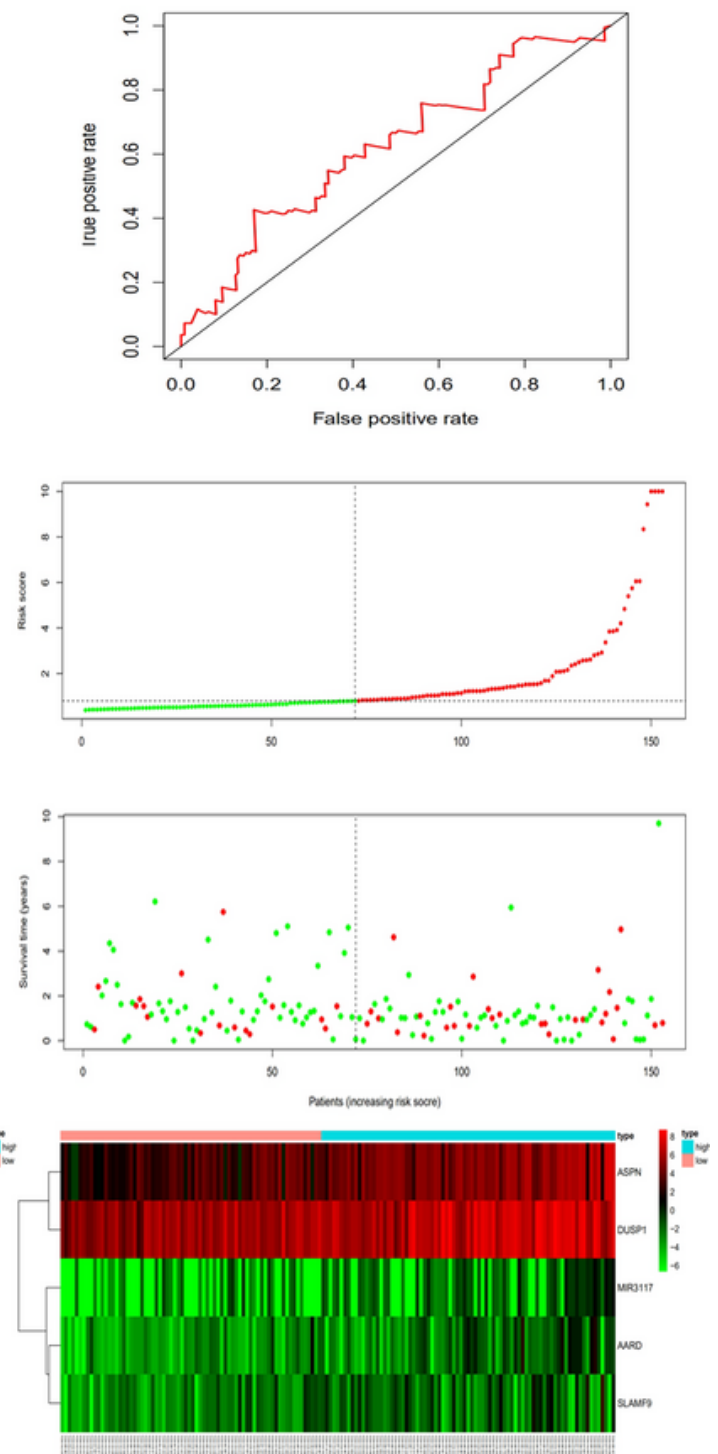

Figure 7

Risk score, heatmap of mRNA expression, time-dependent ROC analysis, and Kaplan-Meier curve of the 5gene signature in the training set and testing set. A, Kaplan-Meier curve of the 5-gene signature. B, Timedependent ROC analysis. C, Risk score, heatmap of mRNA expression. AUC, area under the ROC curves; ROC, receiver operating characteristic. 